Plant Tissue Cult. \& Biotech. 17(1): 97-102, 2007 (June)

\title{
Transient GUS Expression in Decapitated Lentil Embryos
}

\section{Fathi Hassan, M. Imdadul Hoque1, Heiko Kiesecker and Hans-Jörg Jacobsen}

Department of Molecular Genetics, University of Hannover, Herrenhauser Str. 2, 30419

Hannover, Germany

Key words : Transient, GUS expression, Lentil embryos

Lentil (Lens culinaris Medik.) is an important protein- and carbohydrate-rich food for many developing countries and is also popular in developed countries where they are perceived as a healthy component of the diet. Lentil seeds contain about $24.2 \%$ high quality protein, $60 \%$ carbohydrates and $2.4-4.2 \%$ mineral matter (Hulse 1994). Although lentil is one of the oldest cultivated crop plants, its agronomic performance is not better than a semi-domesticated species. The main constraints for lentil breeding and production are poor yield stability and high susceptibility to fungal diseases. Due to the rather narrow genetic base and lack of resistance genes against major fungal diseases in lentil conventional breeding methods cannot be applied for addressing these objectives. In this context the genetic transformation could be used to develop disease resistant plants.

The grain legumes have been less amenable to manipulation in vitro (McClean and Grafton 1989), and generally are recalcitrant to transformation (DeKathen and Jacobsen 1990). Lentil is susceptible to tumor induction by Agrobacterium tumefaciens (Warkentin and McHughen 1991). Although lentil transformation using Agrobacterium (Warkentin and McHughen 1992, 1993) and particle gun bombardment have been reported (Öktem et al. 1999) there is no convincing report on an efficient and stable transformation system for this crop. The susceptibility of different lentil explants to various strains of Agrobacterium has been studied through transient GUS expression.

Four lentil lines, ILL6994, ILL5883, ILL7201 and ILL7012 were obtained from the International Center for Agricultural Research in the Dry Areas (ICARDA), Aleppo, Syria and used in the present investigation. Seeds were soaked in $70 \%$ ethanol for one min and then surface sterilized with $6.0 \%$ commercial bleach for $5 \mathrm{~min}$ and afterwards washed three - four times with sterilized distilled water. The surface sterilized seeds were cultured on water-agar medium and kept in dark at $21 \pm 2^{\circ} \mathrm{C}$ until their germination.

\footnotetext{
${ }^{1}$ Department of Botany, University of Dhaka, Dhaka-1000, Bangladesh.
} 
Leaf segment (LS), shoot tips (ST), epicotyl (EC), cotyledonary nodes (CN), decapitated embryo and half embryo explants were used. Leaf segment, shoot tips, epicotyl, cotyledonary nodes were excised from aseptically grown threeday-old seedlings. For culture of decapitated and half embryos, sprouted seeds were split open and both root and shoot parts of the mature zygotic embryos were cut to obtain decapitated embryo explants. For half-embryo explant, the testa was removed and the embryo was divided into two halves longitudinally with each half attached to one cotyledon.

MS, MSB (macro- and micro-salts of MS and vitamins of B5) and B5 (Gamborg et al. 1968) media were used. The $\mathrm{pH}$ of the medium was adjusted to 5.8 before autoclaving. Filter-sterilized hormone supplements were added to the medium before dispensing it into plastic Petri dishes $(9 \mathrm{~cm}$ dia) or into plastic boxes (10 cm dia).

Two strains of Agrobacterium EHA101 and LBA4404 carrying the disarmed plasmid pIBGUS with GUS reporter gene were used. $250 \mu \mathrm{l}$ A. tumefaciens suspension was taken from the glycerol stock and inoculated into $100 \mathrm{ml}$ Erlenmeyer flask containing $25 \mathrm{ml}$ of YEP medium and antibiotics, i.e. $50 \mathrm{mg} / \mathrm{l}$ kanamycin and $40 \mathrm{mg} / \mathrm{l}$ gentamycin for pIBGUS plasmid. Inoculated flasks were incubated on a rotary shaker for $15 \mathrm{~h}$ at $250 \mathrm{rpm}$ in the dark at $28^{\circ} \mathrm{C}$.

Bacteria were harvested after $15 \mathrm{~h}$ by centrifugation at $4400 \mathrm{rpm}$ for $10-12$ min. The supernatant was discarded and the pellet was resuspended in liquid MSB medium supplemented with either $3.24 \mu \mathrm{M}$ BAP or $5.0 \mu \mathrm{M}$ TDZ. O.D of the bacterial suspension was adjusted to $1.0-1.3$ at $600 \mathrm{~nm}$ using a spectrophotometer with liquid MSB medium. To accelerate the transformation efficiency, acetosyringone was added to the bacterial suspension at a concentration of 100 $\mu \mathrm{M}$. The explants were immersed in Agrobacterium suspension for $1 \mathrm{~h}$. The explants were then placed on a filter paper or directly on to the co-culture medium (MSB semi solid medium supplemented with $3.24 \mu \mathrm{M}$ BAP or $5.0 \mu \mathrm{M}$ TDZ) in darkness for three days at $21^{\circ} \mathrm{C}$.

After co-culture, the explants were washed 2 - 3 times with sterile distilled water followed by immersion in a solution consisting of $100 \mathrm{mM}$ sodium phosphate buffer ( $\mathrm{pH} 7.0$ ), $0.5 \mathrm{mM}$ potassium ferrocyanide and $10 \mathrm{mM}$ EDTA supplemented with $1 \mathrm{mM}(0.5 \mathrm{mg} / \mathrm{ml})$ X-GLUC. Explants were incubated for 4 $5 \mathrm{~h}$ at $37^{\circ} \mathrm{C}$; the buffer was decanted and the explants were rinsed with $70 \%$ ethanol. Explants were then viewed under microscope for GUS-expression.

In this experiment about 1500 explants were used in three replicates for testing the differences between lines/variety and treatments and two replicates for the differences between the Agrobacterium strains. SAS software was used for statistical analysis followed by multiple comparison using LSD at $\alpha=0.05$ (SAS 2000). 
There was hardly any GUS expression in shoot tips, cotyledonary nodes and epicotyl explants; therefore, the most responsive explants, viz. decapitated embryo and half embryo attached with cotyledon explants have been described.

Significant differences in GUS expression were observed with respect to Agrobacterium strain and explants. This is in agreement with other studies (Warkentin and McHughen 1992, Oktem et al. 1999). GUS expression increased when a filter paper was used on the media during co-culture, although not at a significant level (cf. ANOVA at the 0.05 level, where $p=0.0658$ ) (Fig. 1). Kiesecker (2000) observed a similar response for chickpea transformation.

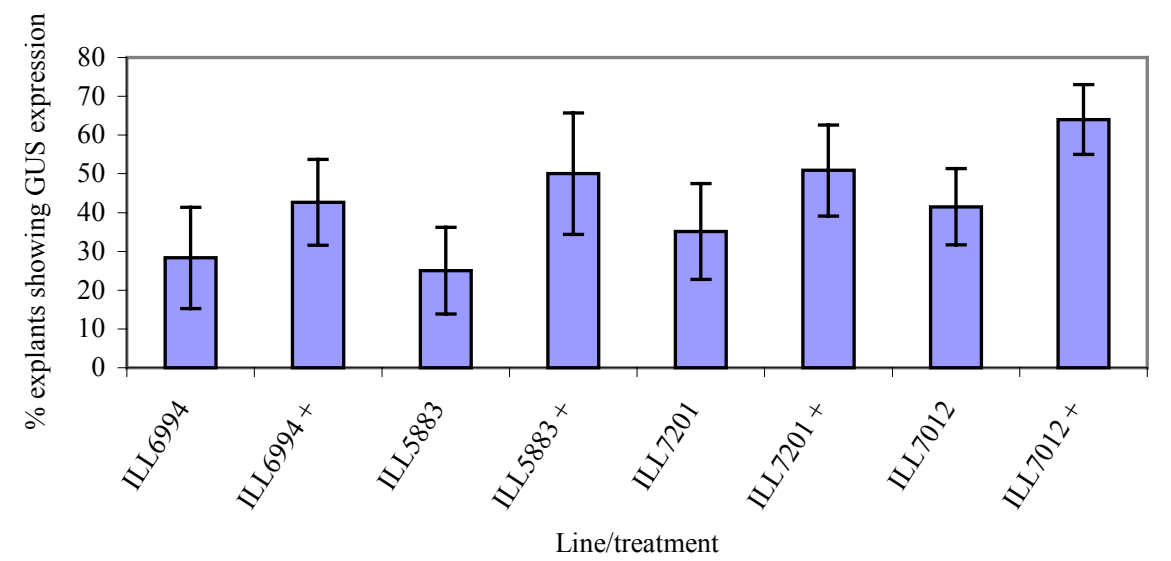

Fig. 1. Effects of Agrobacterium strain EHA101-pIBGUS on GUS gene expression in half embryo attached cotyledon (HE) explants of four lines of lentil, (ILL6994; ILL5883; ILL7201; ILL7012 ' +' indicates use of filter paper on the medium during co-culture). Error bars represent standard error of the mean.

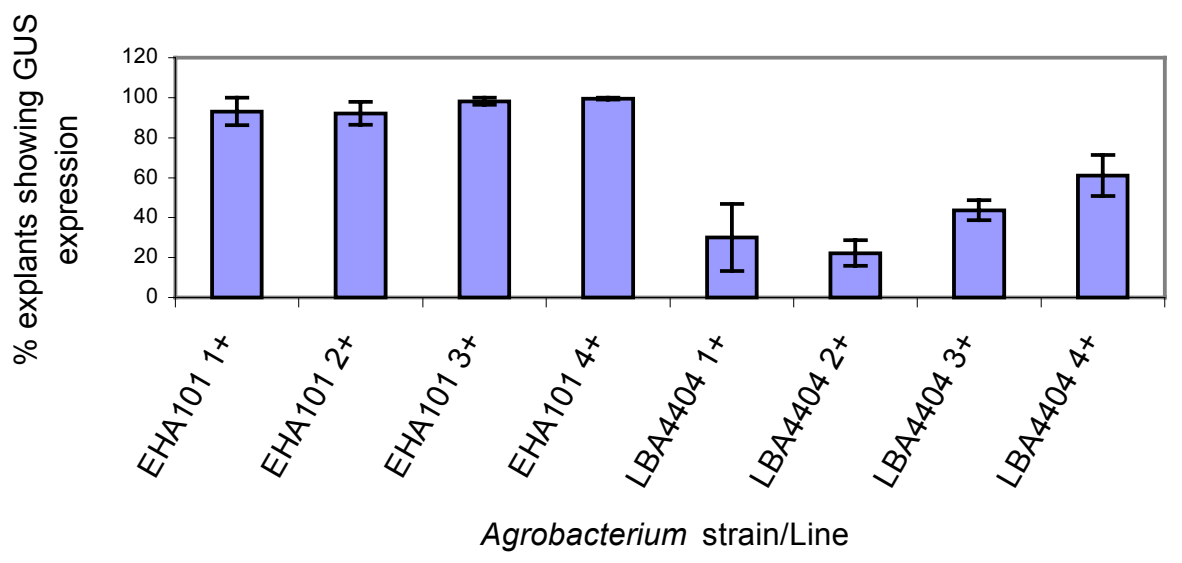

Fig. 2. Effects of Agrobacterium EHA101 and LBA4404 on GUS expression in decapitated embryo explants of four lines of lentil (ILL6994; ILL5883; ILL7201; ILL7012). Error bars represent standard error of the mean. 
Among the four genotypes, ILL7012 line showed the highest GUS expression, although this was not significant by ANOVA at 0.05 level $(p=0.0867)$ (Figs. 1 and 3). The variation in expression may have several reasons: a different T- DNA copy number, cis-acting elements such as silencers and enhancers in the T- DNA target sites, transcriptional interference of T- DNA and target expression units and the general chromatin structure (Warkentin and McHughen 1991).
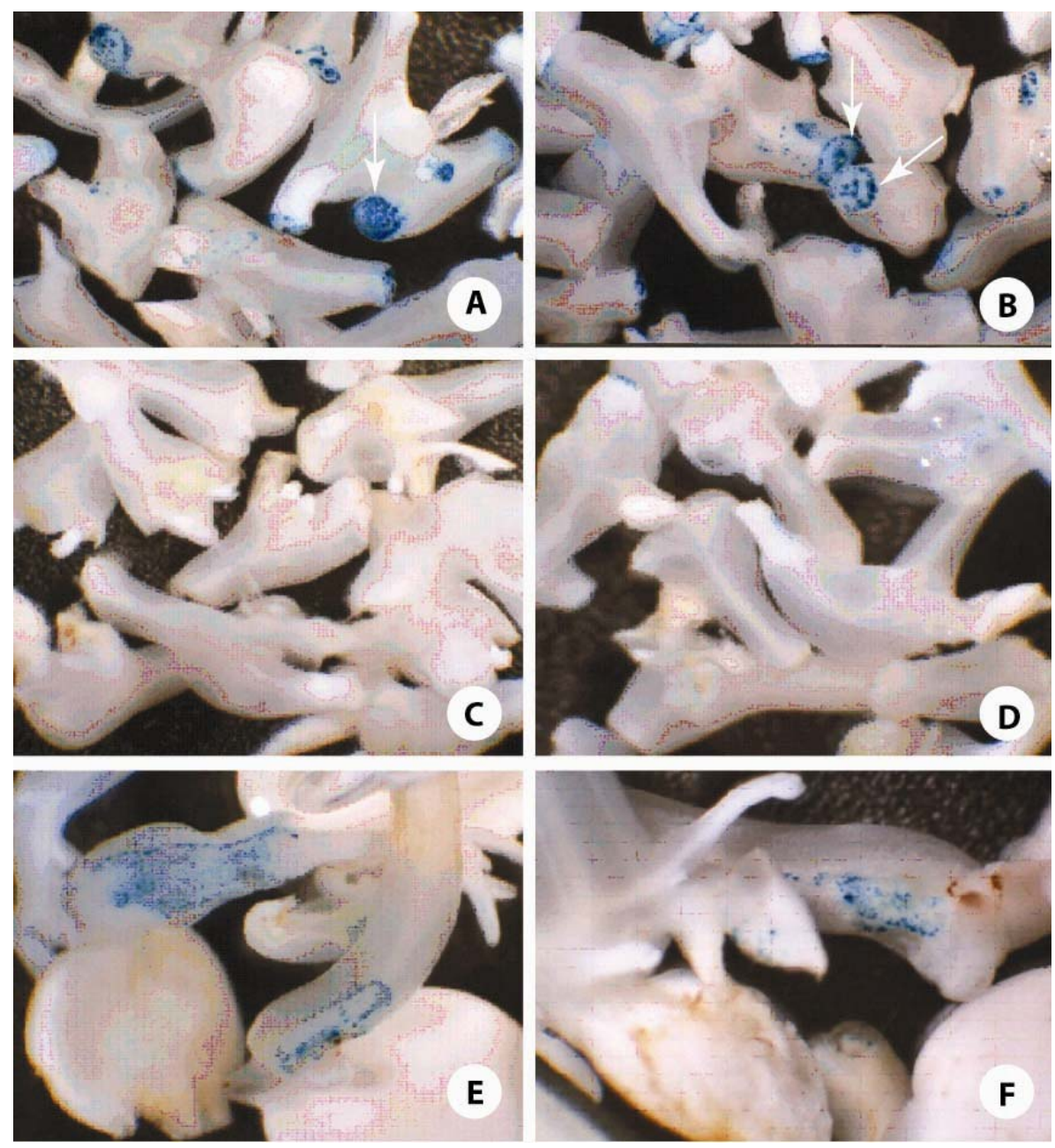

Fig. 3. Effects of Agrobacterium strains on transient GUS-expression in decapitated embryos (A-D) and cotyledon attached-half embryo explant (E \& F) of six lentil lines (A) ILL5883 with EHA101pIBGUS, (B) ILL7012 with EHA101-pIBGUS, (C) ILL5883 with LBA4404-pIBGUS, (D) ILL7012 with LBA4404-pIBGUS (Note: arrows point to the high GUS expression on the cutting sides), (E) ILL6994 with EHA101- pIBGUS, and (F) ILL7012 with EHA101- pIBGUS)

EHA 101 showed maximum GUS expression compared to LBA 4404 and the difference between the two strains was significant at 0.05 level $(p<0.0001)$ (Figs. 2 and 3). Decapitated embryos showed the best response for transient GUS 
expression and a significant difference was observed between the two explants at 0.05 level $(\mathrm{p}<0.0001)$. The highest intensity of GUS expression was observed at the cutting side of the explants (Fig. 3).

Transient GUS assays allow the monitoring of gene expression shortly after transformation. The intron-containing construct is useful for evaluating the expression at an early stage after inoculation, without interfering the activity from contaminating Agrobacteria. Warkentin and McHughen (1992) tested shoot apex, epicotyl and root explants of lentil for GUS expression. They obtained a maximum of $69 \%$ GUS expression from shoot apex explants, having only $1.57 \%$ expression area when co-cultured for seven days. Warkentin and McHughen (1993) again used the cotyledonary nodal explants to see their potentiality for Agrobacterium-mediated genetic transformation. Although there were blue spots in some small buds, they could not recover any transgenic shoot. Öktem et al. (1999) performed transformation experiment with cotyledonary nodes for the expression of GUS gene through Particle Gun Bombardment. Almost 50\% of the bombarded explants expressed the GUS gene; they also claimed that the shoots from the bombarded explants exhibited patches of GUS staining. The decapitated embryo explants used in the present investigation showed more than $90 \%$ transient GUS expression. Previous workers have not used decapitated embryo explants for transformation experiments in lentil (Warkentin and McHughen 1992, 1993, Öktem et al. 1999). Since selection of explants is one of the major steps of any tranformation experiments our results of transient GUS expression, suggests that decapitated embryos can be used as explants for the development of transgenic lines of lentil.

\section{Acknowledgements}

Authors would like to thank Dr. W.Erskine and Dr. M. Baum (ICARDA) for providing the plant material used in this study. First author is grateful to DAAD for providing scholarship to obtain M.Sc degree at Hannover University. The second author (MIH) is indebted to Alexander von Humboldt Stiftung for offering him Georg Forster fellowship to carry out this work.

\section{References}

DeKathen A and Jacobsen HJ (1990) Agrobacterium tumefacien-mediated transformation of Pisum sativum L. using binary and cointegrate vectors. Plant Cell Report 9: 276-279.

Hulse JH (1994) Nature, composition and utilization of food legumes. In: Muehlbauer, FJ Kaiser, W.J. (Eds.). Expanding the production and use of cool season food legumes. Kluwer Academic publishers- The Netherlands. p. 77-97.

Kiesecker H (2000) “Entwicklung eines Agrobacterium tumefaciens vermittelten Gentransfersystems für Kichererbsen (Cicer arietinum L.) - Grundlagen zur gentechnischen Resistenzvermittlung gegenüber Ascochyta rabiei" Dissertation 2000, Universität Hannover, Fachbereich Biologie. 
McClean P and Grafton KF (1989) Regeneration of dry bean (Phaseolus vulgaris L.) via organogenesis. Plant Sci. 60: 117-122.

Öktem HA, Mahmoudian M, Eyidooan F and Yücel M (1999) GUS gene delivery and expression in lentil cotyledonary nodes using particle bombardment. Lens Newsletter 26(1\&2): 3-6.

SAS Institute. (2002) Statistical analysis system, version 8.1, U.S.A.

Warkentin TD and McHughen A (1991) Crown gall transformation of lentil (Lens culinaris Medik.) with virulent strains of Agrobacterium tumefaciens. Plant Cell Reports. 10: 489493.

Warkentin TD and McHughen A (1992) Agrobacterium tutumefaciens-mediated betaglucuronidase (GUS) gene expression in lentil (Lens culinaris Medik.) tissues. Plant Cell Reports. 11: 274-278.

Warkentin TD and McHughen A (1993) Regeneration from lentil cotyledonary nodes and potential of this explant for transformation by Agrobacterium tumefaciens. Lens Newsletter. 20: 26-28. 